\title{
Caracterização bioclimática de sistemas ao ar livre e confinado para a criação de matrizes suínas gestantes
}

\author{
Aérica C. Nazareno ${ }^{1}$, Iran J. 0. da Silva ${ }^{1}$, Maria L. A. N unes ${ }^{1}$, \\ Ariane C. de Castro ${ }^{1}$, Késia 0. S. Miranda ${ }^{1} \&$ Aldie Trabachini ${ }^{1}$
}

\section{RESU M O}

O confinamento de matrizes suínas foi criado com o intuito de maximizar a produtividade; entretanto, existem problemas relacionados ao bem-estar animal. O bjetivou-se avaliar a criação de matrizes suínas gestantes no sistema de confinamento e ao ar livre, com relação ao ambiente térmico e às respostas fisiológicas. 0 experimento foi realizado em Monte Mor/SP. A avaliação fisiológica foi realizada por meio do registro das variáveis: frequência respiratória e temperatura de pele. Foram registradas as variáveis meteorológicas: temperatura de bulbo seco, temperatura de bulbo úmido e temperatura de globo negro, caracterizando o ambiente por meio da entalpia e índice de temperatura de globo e umidade. Foram utilizados seis animais por tratamento. 0 delineamento experimental foi inteiramente casualizado em parcelas subdivididas, e as médias comparadas pelo teste de Tukey. As variáveis fisiológicas e meteorológicas apresentaram valores superiores no confinamento. 0 sistema de criação ao ar livre potencializou as trocas térmicas entre os animais e o ambiente, o que refletiu em menor estresse por calor observado nos animais.

Palavras-chave: suíno, bem-estar animal, conforto térmico, sistemas de produção

\section{Bioclimatic characterization of outdoor and confined systems for pregnant sows}

\begin{abstract}
Pregnant sows confinement systems were created in order to maximize the productivity, however there are problems concerning the animal welfare. The aim of this research was to evaluate pregnant sows in outdoors and in confinement systems in relation to the thermal environment and physiological animal responses. The experiment was conducted in a commercial farm in Monte Mor city, São Paulo, Brazil. The physiological evaluation was performed by recording physiological variables, such as respiratory frequency and skin temperature. Furthermore, variables like dry bulb temperature, wet bulb temperature, and black globe temperature were also evaluated to characterize the ambient by means of enthalpy and black globe humidity index. In each treatment six animals were evaluated. The experimental design was completely randomized in a split-plot version whose averages were compared by the Tukey test. The findings of the experiment revealed higher values for all the bioclimatic variables in the confined treatment. Hence, the outdoor system provided better thermal conditioning for pigs, resulting in a lower heat stress.
\end{abstract}

Key words: swine, animal welfare, thermal comfort, livestock 


\section{INTRODUÇÃO}

O sistema de criação confinado foi criado com o intuito de reduzir o trabalho e a perda energética dos animais, ganhar espaço e melhorar o controle ambiental. No entanto, os problemas de bem-estar animal são agravados (Pandorfi et al., 2008; Nazareno et al., 2009), uma vez que a condição imposta restringe o comportamento natural dos animal.

O sistema predominantemente utilizado para a criação de matrizes suínas é, em sua maioria, realizado em confinamento total, utilizando-se gaiolas ou baias coletivas, alternativas que impactam negativamente no bem-estar desses animais. A utilização de gaiolas implica na falta de contato social e na incapacidade desses animais realizarem exercícios e entrarem em contato com estímulos ambientais (Barnett et al., 2001). Por outro lado, a criação em baias coletivas também possui controvérsias em relação ao bem-estar animal uma vez que, normalmente, o ambiente não possui enriquecimento ambiental e existe alta incidência de agressividade entre os animais (Remience et al., 2008).

O sistema de criação de suínos ao ar livre apresenta diversas vantagens em relação ao sistema confinado, como baixo investimento inicial (Rachuonyo et al., 2002; Miao et al., 2004), menor produção de odores indesejáveis, melhores condições ambientais (Rachuonyo et al., 2002) e melhoria nas condições de bem-estar dos animais (Baxter et al., 2009). Além dessas vantagens e caso sejam adotados manejos adequados, a criação ao ar livre permitirá complementar a dieta dos animais com o uso da pastagem, proporcionando maior fertilidade de solo e propriedades físicas desejáveis, tal como diminuição da erosão e aumento da ciclagem de nutrientes (Rodríguez-Estévez et al., 2009).

Independente do sistema de criação adotado, o ambiente deve ser analisado do ponto de vista de conforto térmico e bem-estar animal, uma vez que referidos fatores afetam diretamente as condições de manutenção do balanço térmico e a produtividade animal. O suíno tem dificuldade para dissipar calor em ambiente de alta temperatura e umidade, devido, sobremaneira, ao fato desses animais serem inábeis em suar (Barnett et al., 2001), além de evolutivamente adaptados a climas temperados (Bloemhof et al., 2008).

A zona termoneutra representa o ambiente térmico ótimo no qual os suínos possuem condições adequadas para uma produção melhor. Para as matrizes suínas a temperatura ambiente considerada de conforto varia entre 7 e $23{ }^{\circ} \mathrm{C}$ (Noblet et al., 1989; Lucas \& Cruz, 1997); entretanto, altas temperaturas no interior das instalações influenciam negativamente a eficiência reprodutiva, com redução da fertilidade (Peltoniemi et al., 1999), em especial em fêmeas primíparas, além de atraso da maturidade sexual (Flores et al., 1989; Perez \& Gutierez, 1987) e maior mobilização de gordura corporal durante a lactação (Barbet al., 1991).

Em condições de estresse por calor os animais aumentam as perdas de calor evaporativas, sobretudo pelo aumento da frequência respiratória (Huynh et al., 2006). Além da frequência respiratória a temperatura da pele é uma variável fisiológica que pode ser alterada em condições de estresse por calor. $\mathrm{O}$ aumento da temperatura da pele se deve à intensificação da circulação periférica, como forma de dissipação de calor (Black et al., 1993; Williams, 2009).

Em condições de clima e realidade produtiva no Brasil, a análise da criação de matrizes suínas ao ar livre é carente em termos de informações bioclimáticas, ante o que se objetivou avaliar a criação de matrizes suínas gestantes no sistema de confinamento e ao ar livre, com relação ao ambiente térmico e às respostas fisiológicas.

\section{Material E MÉTODOS}

O experimento foi desenvolvido durante o mês de agosto de 2009, em uma granja comercial de produção de suínos, localizada no município de Monte Mor, SP, situada a 22³3' S de latitude e $47^{\circ} 11^{\prime}$ W de longitude e altitude média de $560 \mathrm{~m}$. O clima da região é caracterizado como mesotérmico Cwa (tropical de altitude), com temperatura do mês mais frio entre 3 e $18^{\circ} \mathrm{C}$, com inverno seco e temperatura do mês mais quente maior que $22{ }^{\circ} \mathrm{C}$, segundo classificação de Köppen (Pereira et al., 2002).

Foram avaliados dois sistemas de criação, considerados neste trabalho como tratamentos: ar livre (AL) e confinado (CONF). As fêmeas foram alojadas nos tratamentos a partir do $30^{\circ}$ dia de gestação, após permanecerem em ambiente de gaiola durante o início da gestação. A instalação utilizada para o tratamento CONF é caracterizada, tipologicamente, por $60 \mathrm{~m}$ de comprimento por $15 \mathrm{~m}$ de largura, pé-direito de $2,3 \mathrm{~m}$, com orientação leste-oeste. A cobertura é de telhas de fibrocimento, sem forro de revestimento, apresentando fechamento lateral em alvenaria, com mureta de $1,10 \mathrm{~m}$. No tratamento CONF as fêmeas foram alojadas em uma baia coletiva de $6 \mathrm{~m}$ de comprimento por $3 \mathrm{~m}$ de largura e muretas de contenção de $1,10 \mathrm{~m}$ de altura, com ripado de bambu no terço final, de maneira a garantir sombreamento aos animais. O piso da baia era de concreto, totalizando uma área livre de $18 \mathrm{~m}^{2}$, dispondo de $3 \mathrm{~m}^{2}$ por animal.

Para o tratamento AL foi utilizado um piquete de $15 \mathrm{~m}$ de comprimento por $30 \mathrm{~m}$ de largura, totalizando uma área livre de $450 \mathrm{~m}^{2}$, dispondo de $75 \mathrm{~m}^{2}$ por animal, formado por gramínea estrela africana (Cynodon plesctostachyum) e cercado eletrificado. Os animais tinham a disponibilidade de sombreamento artificial (ripado de bambu).

Foram utilizados seis animais por tratamento de linhagens comerciais (CB25 e CB23 Agroceres e Dalland Topigs). A alimentação foi feita com ração à base de milho e sorgo, com fornecimento de $3250 \mathrm{kcal} \mathrm{EM} \mathrm{dia}^{-1}$ e distribuída na base de 2,2 $\mathrm{kg} \operatorname{dia}^{-1}$, durante o período de gestação.

A avaliação térmica ambiental foi realizada por meio do registro dos dados meteorológicos, nos dois sistemas de criação (AL e CONF) cujas variáveis meteorológicas registradas foram: temperatura de bulbo seco $\left(\mathrm{Tbs},{ }^{\circ} \mathrm{C}\right)$, temperatura de bulbo úmido ( $\mathrm{Tbu},{ }^{\circ} \mathrm{C}$ ) e temperatura de globo negro $\left(\mathrm{Tg},{ }^{\circ} \mathrm{C}\right) \mathrm{o}$ que permitiu a caracterização da eficiência térmica nos diferentes sistemas de criação.

Realizaram-se os registros das variáveis meteorológicas em intervalos de $1 \mathrm{~h}$, entre as $7 \mathrm{~h} 45 \mathrm{~min}$ e as $16 \mathrm{~h} 45 \mathrm{~min}$, durante 8 dias do ciclo de produção. As variáveis Tbs e Tbu foram registradas por meio de um termohigrômetro da marca 
Incoterm ${ }^{\circledR}$, escala entre -10 e $50{ }^{\circ} \mathrm{C}$, limite de erro de $\pm 1{ }^{\circ} \mathrm{C}$. A temperatura de globo negro foi registrada com auxílio de um termômetro comum $\left(-20 \mathrm{a} 110^{\circ} \mathrm{C}\right)$ acoplado a uma esfera oca de polietileno de alta densidade, pintada de preto fosco. A velocidade do vento foi registrada com auxílio de um anemômetro de hélice digital da marca thermo anemometer®. Os equipamentos foram instalados nos dois sistemas de produção, a uma altura de 1,30 $\mathrm{m}$ do piso, representando o microclima do local. No sistema CONF os equipamentos foram instalados no centro geométrico da baia e no sistema AL em uma área coberta com telhas de fibrocimento, localizada na área de alimentação do piquete.

Realizou-se a determinação da eficiência térmica das instalações através dos dados referentes às variáveis meteorológicas registradas nos ambientes estudados, em que se determinaram o índice de temperatura de globo e a umidade (ITGU) (Buffington et al. 1981) e entalpia (h) (Albright, 1990), conforme equações abaixo:

$$
\text { ITGU }=\text { Tg }+0,36 \mathrm{Tpo}-330,08
$$

em que:

$\mathrm{Tg}$ - temperatura de globo negro, $\mathrm{K}$

Tpo - temperatura de ponto de orvalho, $\mathrm{K}$

$$
\mathrm{h}=1,006 \mathrm{Tbs}+\mathrm{W}(2501+1,805 \mathrm{Tbs})
$$

em que:

Tbs- temperatura de bulbo seco, ${ }^{\circ} \mathrm{C}$

W - razão de mistura, $\mathrm{kg}$ vapor d'água $\mathrm{kg}^{-1}$ ar seco

$$
\mathrm{W}=(0,622 \mathrm{ea}) /\left(\mathrm{P}_{\mathrm{atm}}-\mathrm{ea}\right)
$$

em que:

ea - pressão atual de vapor d'água, $\mathrm{kPa}$

Patm - pressão atmosférica, $\mathrm{kPa}$

Para avaliação das variáveis fisiológicas registraram-se os dados de temperatura de pele (TP), nas regiões paleta, pernil, nuca e lombo e de frequência respiratória (FR) dos suínos (mov. $\min ^{-1}$ ). A determinação dessas variáveis foi realizada em intervalos de 4 h, às 9:00, 13:00 e 16:00 h, em três dias, nos 6 animais de cada tratamento. A verificação da frequência respiratória ( $\mathrm{mov} \mathrm{min}^{-1}$ ) se deu a partir da contagem do número de movimentos abdominais realizados pelo suíno, pelo tempo de 1 min. Para medição da temperatura de pele $\left({ }^{\circ} \mathrm{C}\right)$ utilizou-se termômetro de infravermelho da marca Icell ${ }^{\circledR}$.
O delineamento experimental para as variáveis ambientais foi o inteiramente casualizado (DIC) com parcelas subdividas em que os sistemas de criação (AL e CONF) foram alocados nas parcelas e os dias nas subparcelas. Para os parâmetros fisiológicos (frequência respiratória e temperatura de pele), adotou-se o delineamento em faixa com parcelas subsubdivididas inteiramente casualizadas (DIC) sendo os sistemas de criação alocados nas parcelas, os dias em subparcelas e os horários em sub-subparcelas, com 6 repetições, utilizando-se 6 suínos por tratamento, totalizando 12 suínos. Para comparação das médias utilizou-se o teste de Tukey a nível de 5\% de probabilidade, com o auxílio do programa estatístico Statistical Analysis System (SAS, 2008).

\section{RESULTADOS E DISCUSSÃO}

Os sistemas de criação apresentaram diferença significativa para Tbs (Tabela 1), verificando-se maior temperatura no sistema de criação CONF, principalmente em virtude da maior taxa de lotação e aporte de energia gerada pelos animais neste tratamento. Os valores médios de Tbs foram de 24,34 e 22,75 ${ }^{\circ} \mathrm{C}$, para os tratamentos $\mathrm{CONF}$ e $\mathrm{AL}$, respectivamente.

Com relação à umidade relativa do ar, observou-se o maior valor médio no tratamento CONF com diferença significativa entre os tratamentos, obtendo-se os valores 56,31 e 53,07\%, para CONF e AL, respectivamente.

Referente aos valores de entalpia do ar nota-se que o maior valor médio registrado nos diferentes sistemas de criação foi detectado no tratamento CONF, diferindo significativamente $(\mathrm{P}<0,05)$ do tratamento AL, com valores da ordem de 49,84 e $47,87 \mathrm{~kJ} \mathrm{~kg}^{-1}$ de ar seco, respectivamente. Tais valores representam a quantidade de energia contida nos sistemas avaliados, levando-se em consideração a Tbs e a razão de mistura (kg de vapor d' água $\mathrm{kg}^{-1} \mathrm{de}$ ar seco), sendo assim, um bom indicador da condição de conforto proporcionada aos animais.

A variável ITGU apresentou diferença significativa entre os tratamentos, com maior valor no tratamento CONF (74,25 e 72,28 , respectivamente). Os maiores valores de ITGU no tratamento CONF em relação ao tratamento AL evidenciam condições climáticas mais críticas no ambiente confinado, uma vez que este índice leva em consideração o efeito conjugado da temperatura de ponto de orvalho e temperatura de globo negro.

Os dados médios de Tbs mostram que as porcas no tratamento AL permaneceram em condições mais próximas da condição de conforto indicada por Noblet et al. (1989). A Figura

\begin{tabular}{|c|c|c|c|c|c|}
\hline Sistema de criação & Tbs $\left({ }^{\circ} \mathrm{C}\right)$ & $\operatorname{Tg}\left({ }^{\circ} \mathrm{C}\right)$ & UR (\%) & $\mathrm{h}\left(\mathrm{kJ} \mathrm{kg} \mathbf{~}^{-1}\right)$ & ITGU \\
\hline $\begin{array}{l}\text { AL } \\
\text { CONF }\end{array}$ & $\begin{array}{l}22,75 b \pm 3,58 \\
24,34 a \pm 4,37\end{array}$ & $\begin{array}{l}26,43 b \pm 3,82 \\
28,49 a \pm 7,22\end{array}$ & $\begin{array}{l}53,07 b \pm 19,89 \\
56,31 a \pm 19,23\end{array}$ & $\begin{array}{l}47,87 b \pm 7,11 \\
49,84 a \pm 5,41 \\
\end{array}$ & $\begin{array}{l}72,28 b \pm 3,58 \\
74,25 a \pm 6,74\end{array}$ \\
\hline $\begin{array}{l}\text { CV \% } \\
\text { Teste F }\end{array}$ & $\begin{array}{c}8,54 \\
15,74 *\end{array}$ & $\begin{array}{c}14,74 \\
9,12 *\end{array}$ & $\begin{array}{l}17,62 \\
15,96^{*}\end{array}$ & $\begin{array}{l}9,46 \\
5,32 *\end{array}$ & $\begin{array}{c}4,28 \\
10,79 *\end{array}$ \\
\hline
\end{tabular}

Tabela 1. Valores médios e desvio padrão das variáveis ambientais para os sistemas de criação ao ar livre (AL) e confinamento total (CONF)

Na mesma coluna médias seguidas da mesma letra não diferem entre si a nível de $5 \%$ de probabilidade pelo teste de Tukey. Tbs - temperatura de bulbo seco, Tg - temperatura de globo negro, UR umidade relativa, $\mathrm{h}$ - entalpia e ITGU - índice de temperatura de globo e umidade, NS - não significativo, ${ }^{*}$ - significativo a $5 \%(P<0,05)$ 
1A mostra que, independentemente, do horário de avaliação, a temperatura no tratamento CONF esteve acima quando comparada com a do tratamento AL. Este fato evidencia melhores condições de conforto proporcionado aos animais criados ao ar livre.
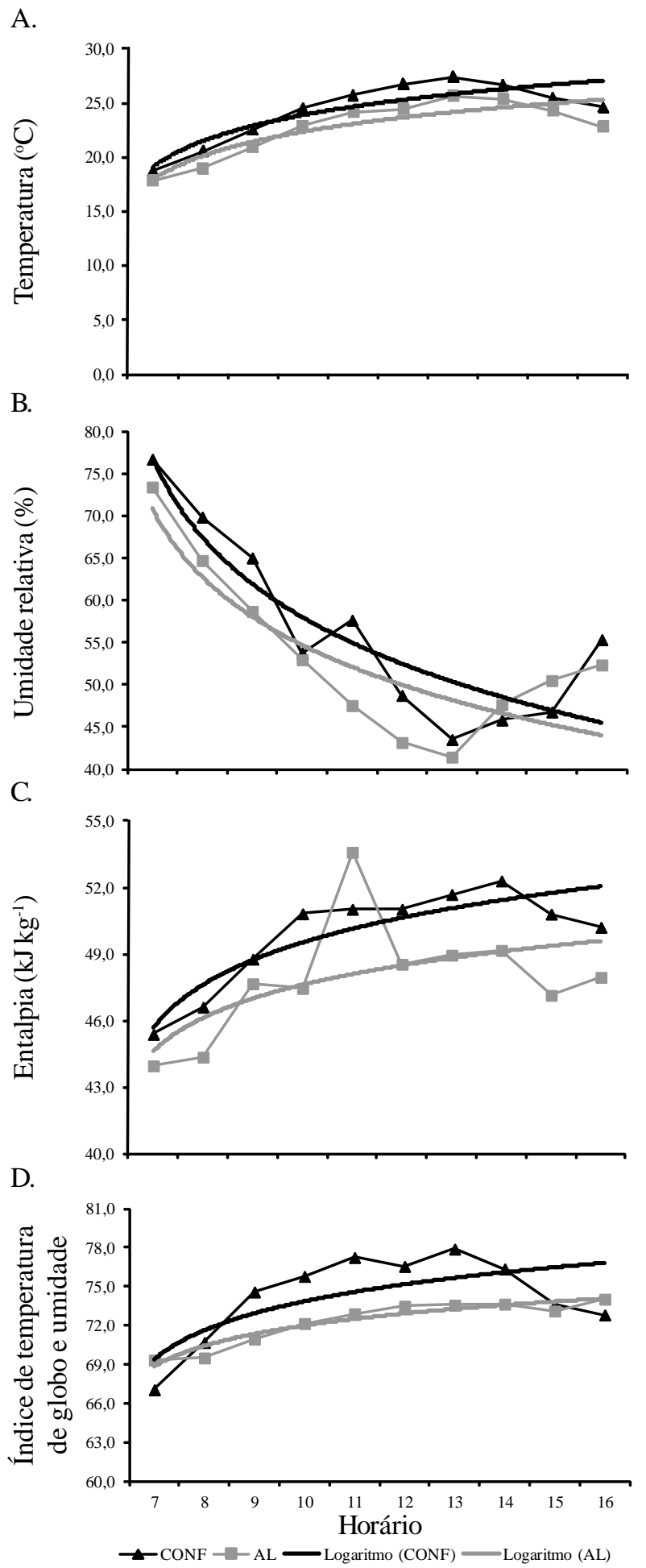

Figura 1. Variação média da temperatura $(A)$, umidade relativa (B), ental pia (C) e índice de temperatura de globo de umidade (D) em diferentes horários submetidos ao sistema de criação ao ar livre $(A L)$ e confinamento total (CONF)
Os valores de temperatura começaram a aumentar a partir das $9 \mathrm{~h}$, chegando a valores máximos às $13 \mathrm{~h}$, que permaneceram elevados até aproximadamente as $16 \mathrm{~h}$. Costa (2009) menciona que os horários de temperatura mais crítica ocorrem das $11 \mathrm{~h}$ às $15 \mathrm{~h}$.

Nota-se, no decorrer do período avaliado que a temperatura média diária no tratamento CONF permaneceu, durante a maior parte do tempo, superior aos $23{ }^{\circ} \mathrm{C}$, caracterizando-se como situação de alerta, o que pode causar problemas reprodutivos, sobremaneira em fêmeas primíparas (Peltoniemi et al., 1999; Nunes et al., 2003). Entretanto, o sistema de criação AL se manteve dentro da faixa de conforto térmico, de acordo com os limites propostos por Noblet et al. (1989).

Na Figura 1B são apresentados os valores médios de umidade relativa do ar que permaneceram na faixa de 53,07 e $56,31 \%$ para os tratamentos AL e CONF, respectivamente, o que representa valores recomendados para matrizes suínas (Moura, 1999).

A variação entálpica nos tratamentos avaliados apresentou valores médios abaixo do recomendado para suínos na fase de gestação que, conforme Moura (1999), varia de 55,4 a 63,0 $\mathrm{kJ} \mathrm{kg}^{-1}$ de ar seco (Figura 1C). Referidos valores de entalpia encontrados em ambos os tratamentos, expressam a quantidade de energia contida em uma mistura de ar seco e vapor d'água influenciando, desta forma, as trocas térmicas entre o animal e o meio.

Os resultados de ITGU são apresentados na Figura 1D, a qual indica que o sistema de criação CONF foi o que obteve maiores valores médios em relação ao AL, durante a maioria dos horários analisados sinalizando, assim, piores condições de conforto térmico. Os horários de menor ITGU foram obtidos no período da manhã e os maiores valores ocorreram entre $10 \mathrm{~h}$ e 15 h, concordando com os resultados de Tolon \& Nääs (2005).

Na Tabela 2 a análise de variância demonstra que ocorreram diferenças estatísticas significativas entre os sistemas de criação para as temperaturas da pele, nas regiões do lombo, paleta e pernil, com maiores valores médios no tratamento CONF.

No tratamento CONF foram obtidos valores médios de 31,92, 32,18 e $32,21^{\circ} \mathrm{C}$, contra $30,02,31,22$ e $30,18^{\circ} \mathrm{C}$ no tratamento $\mathrm{AL}$ para as temperaturas do lombo, paleta e pernil, respectivamente, resultados que evidenciam um estresse térmico maior no sistema confinado, corroborando com resultados obtidos por Edwards (2005), em que animais criados ao ar livre mostraram menor estresse térmico.

A frequência respiratória (FR) apresentou diferença $(\mathrm{P}<$ 0,05) entre os sistemas de criação com valores médios de 31,07

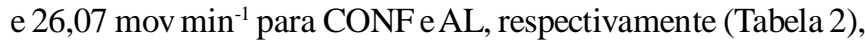
o que mostra sinais de desconforto térmico em ambos os tratamentos. Fêmeas gestantes em condições de conforto térmico apresentam taxa respiratória em torno de 15 a 20 mov $\mathrm{min}^{-1}$. Os movimentos respiratórios podem aumentar de $22 \mathrm{a}$ $100 \%$, em função do ambiente térmico (Nunes et al., 2003; Manno et al., 2005; Silva et al., 2008). Os maiores valores de temperatura no tratamento CONF e os índices avaliados, explicam o fato da FR ter sido maior neste tratamento. 
Tabela 2. Valores médios e desvio padrão das variáveis fisiológicas para os sistemas de criação ao ar livre (AL) e confinamento total (CO F)

\begin{tabular}{|c|c|c|c|c|c|}
\hline \multirow{2}{*}{ Sistema de criação } & \multicolumn{4}{|c|}{ Temperatura $\left({ }^{\circ} \mathrm{C}\right)$} & \multirow{2}{*}{$\begin{array}{l}\text { Frequência respiratória } \\
\qquad \operatorname{mov~min}^{-1}\end{array}$} \\
\hline & Lombo & Paleta & Pernil traseiro & Nuca & \\
\hline $\begin{array}{l}\mathrm{AL} \\
\text { CONF }\end{array}$ & $\begin{array}{l}30,02 b \pm 3,60 \\
31,92 a \pm 3,39 \\
\end{array}$ & $\begin{array}{l}31,22 b \pm 3,18 \\
32,18 a \pm 2,52\end{array}$ & $\begin{array}{l}30,18 b \pm 3,29 \\
32,21 a \pm 2,93\end{array}$ & $\begin{array}{l}31,80 a \pm 3,31 \\
32,15 a \pm 2,77\end{array}$ & $\begin{array}{l}26,07 b \pm 8,18 \\
31,04 a \pm 8,54 \\
\end{array}$ \\
\hline $\begin{array}{l}\text { CV \% } \\
\text { Teste F }\end{array}$ & $\begin{array}{c}8,954689 \\
3,11^{*}\end{array}$ & $\begin{array}{c}5,369517 \\
6,81^{*}\end{array}$ & $\begin{array}{c}6,088909 \\
6,96^{*}\end{array}$ & $\begin{array}{c}6,078305 \\
5,43 \mathrm{NS}\end{array}$ & $\begin{array}{c}29,80656 \\
1,13^{*}\end{array}$ \\
\hline
\end{tabular}

\section{CONCLUSÕES}

1. As variáveis meteorológicas e os índices de conforto térmico ambientais mostram que o sistema de criação ao ar livre permitiu, às matrizes suínas na fase de gestação, melhor condicionamento térmico ambiental.

2. Os parâmetros fisiológicos (frequência respiratória e temperatura de pele) apontaram valores mais adequados para o sistema de criação ao ar livre, como resposta ao menor estresse térmico, atendendo às condições de bem-estar animal.

\section{LITERATURA CITADA}

Albright, L. D. Environment control for animals and plants. St. Joseph: American Society of Agricultural Engineers Michigan. 1990. 453p. ASAE Texbook, 4

Barb, C. R.; Estienne, M. J.; Kraeling, R. R.; Marple, D. N.; Rampacek, G. B.; Rahe, C. H.; Sartin, J. L. Endocrine changes in sows exposed to elevated ambient temperature during lactation. Domestic Animal Endocrinology, v.8, p.117-1127, 1991.

Barnett, J. L.; Hemsworth, P. H.; Cronin, G. M.; Jongman, E. C.; Hutson, G. D. A review of the welfare issues for sows and piglets in relation to housing. Australian Journal of Agricultural Research, v.52, p.1-28, 2001.

Baxter, E. M.; Jarvis, S.; Sherwood, L.; Robson, S. K.; Ormandy, E.; Farish, M.; Smurthwaite, K. M.; Roehe, R.; Lawrence, A. B.; Edwards, S. A. Indicators of piglet survival in an outdoor farrowing system. Livestock Science, v.124, p.266-276, 2009.

Black, J. L.; Mullan, B. P.; Lorschy, M. L.; Giles, L. R. Lactation in the sow during heat stress. Livestock Production Science, v.35, p.153-170, 1993.

Bloemhof, S.; Waaij, E. H. van der; Merks, J. W. M.; Knol, E. F. Sow line differences in heat stress tolerance expressed in reproductive performance traits. Journal of Animal Science, v.86, p.3330-3337, 2008.

Buffington, D. E.; Colazzo-Arocho, A.; Caton, G. H. Black globe humidity comfort index (BGHI) as comfort equation for dairy cows. Transaction of the ASAE, v.24, p.711-714. 1981.

Costa, A. F. G. M. Metodologia em projeto de bloqueador de radiação solar (brises) com estrutura móvel pivotante para conforto ambiental em aviários. Exacta, v.7, p.13-20, 2009.

Edwards, S. A. Product quality attributes associated with outdoor pig production. Livestock Production Science, v.94, p.5-14. 2005.

Flores, B.; Cantley, T. C.; Martin, M. J.; Day, B. N. Effect of elevated ambient temperatures on puberty in gilts. Journal of Animal Science, v.67, p.779-784, 1989.
Huynh, T. T. T.; Aarnink, A. J. A.; Truong, C. T.; Kemp, B.; Verstegen, M. W. A. Effects of tropical climate and water cooling methods on growing pigs' responses. Livestock Science, v.104, p. 278-291, 2006.

Lucas, E. M.; Cruz, V. F. Efeito do clima do alentejo no microclima das instalações para suínos. Revista Portuguesa de Zootecnia, v.4, p.37-52, 1997.

Manno, M. C.; Oliveira, R. F. M.; Donzele, J. L. Ferreira, A. S.; Oliveira, W. P.; Lima, K. R. S.; Vaz, R. G. M. V. Efeito da temperatura ambiente sobre o desempenho de suínos dos 15 aos 30 kg. Revista Brasileira de Zootecnia, v.34, p.19631970, 2005.

Miao, Z. H; Glatz, P. C.; Ru, Y. J. Review of production, husbandry and sustainability of free-range pig production systems. Asian-Australasian Journal of Animal Science. v.17, p.1615-1634, 2004.

Moura, D. J. Ventilação na suinocultura. In: Silva, I. J. O. (ed.) Ambiência e qualidade na produção industrial de suínos. Piracicaba: Fundação de Estudos Agrários Luiz de Queiroz, 1999. p.149-179.

Nazareno, A. C.; Pandorfi, H.; Almeida, G. L. P.; Giongo, P. R.; Pedrosa, E. M. R.; Guiselini, C. Avaliação do conforto térmico e desempenho de frangos de corte sob regime de criação diferenciado. Revista Brasileira de Engenharia Agrícola e Ambiental, v.13, p.802-808, 2009.

Noblet, J.; Dourmad, J. Y.; Dividich, J.; Dubois, S. Effect of ambient temperature and addition of straw or alfafa in the diet on energy metabolism in pregnant sows. Livestock Production Science, v.21, p.309-324, 1989.

Nunes, C. G. V.; Costa, E. P.; Oliveira, R. F. M.; Donzele, J. L.; Nunes, R. V.; Carvalho, G. R. Efeito do acondicionamento térmico ambiental sobre o desempenho reprodutivo da fêmea suína. Revista Brasileira de Zootecnia, v.32, p.854-863, 2003.

Pandorfi, H.; Silva, I. J. O.; Piedade, S. M. S. Conforto térmico para matrizes suínas em fase de gestação, alojadas em baias individuais e coletivas. Revista Brasileira de Engenharia Agrícola e Ambiental, v.12, p.326-332, 2008.

Peltoniemi, O. A. T.; Love, R. J.; Heinomen, M.; Tuovinem, V.; Saloniemi, H. Seasonal and management effects on fertility of the sow: A descriptive study. Animal Reproduction Science, v.55, p.47-61, 1999.

Pereira, A. R.; Angelocci, L. R.; Sentelhas, P. C. Agrometeorologia: Fundamentos e aplicações práticas. Guaíba: Agropecuária, 2002. 478p.

Perez, P. P.; Gutierez, J. F. P. Summer infertility of swine stock in Spain. Luxemburg: Commission of the European Communities, 1987. p.25-28. 
Rachuonyo, H. A.; Pond, W. G.; Mcglone, J. J. Effects of stocking rate and crude protein intake during gestation on ground cover, soil-nitrate concentration, and sow and litter performance in an outdoor swine production system. Journal of Animal Science, p.1451-1461, 2002.

Remience, V.; Wavreille, J.; Canart, B.; Meunier-Salau, N. M. C.; Prunier, A. Bartiaux-Thill, N.; Nicks, B.; Vandenheede, M. Effects of space allowance on the welfare of dry sows kept in dynamic groups and fed with an electronic sow feeder. Applied Animal Behaviour Science, v.112, p.284296, 2008.

Rodríguez-Estévez, V.; García, A.; Peña, F.; Gómez, A. G. Foraging of iberian fattening pigs grazing natural pasture in the dehesa. Livestock Science, v.120, p.135-143, 2009.
SAS - Statistical analysis system: Realease 9.1.3, (software). Cary: Sas Institute, 2008. 620p.

Silva, I. J. O; Pandorfi, H.; Piedade, S. M. S. Influência do sistema de alojamento no comportamento e bem-estar de matrizes suínas em gestação. Revista Brasileira de Zootecnia, v.37, p.1319-1329, 2008.

Silva, R. G. Introdução à bioclimatologia animal. São Paulo: Nobel, 2000, 286p.

Tolon, Y. B.; Nääs, I. A. Avaliação de tipos de ventilação em maternidade de suínos. Engenharia Agrícola, v.25, p.565$574,2005$.

Williams, A. M. Effects of heat stress on reproduction and productivity of primiparous sows and their piglets' performance. Missouri: Universidade de Missouri, 2009, 222p. Dissertação Mestrado 